\title{
Effect of Axon Misdirection on Recovery of Electromyographic Activity and Kinematics after Peripheral Nerve Injury
}

\author{
Manning J. Sabatier ${ }^{a}$ b Bao Ngoc To ${ }^{a}$ Jennifer Nicolini ${ }^{a}$ Arthur W. English ${ }^{a}$ \\ ${ }^{a}$ Department of Cell Biology, Emory University School of Medicine, Atlanta, Ga., and \\ ${ }^{b}$ Department of Natural Sciences, Health and Fitness Management, Clayton State University, Morrow, Ga., USA
}

\section{Key Words}

Sciatic nerve $\cdot$ Soleus $\cdot$ Tibialis anterior $\cdot$ Locomotion

\begin{abstract}
In this study, patterns of activity in the soleus (Sol) and tibialis anterior (TA) muscles and hindlimb kinematics were evaluated during slope walking in rats after transection and surgical repair either of the entire sciatic nerve (Sci group) or of its two branches separately, the tibial and common fibular nerves (T/CF group). With the latter method, axons from the tibial and common fibular nerves could not reinnervate targets of the other nerve branch after injury, reducing the opportunity for misdirection. Activity in the TA shifted from the swing phase in intact rats to nearly the entire step cycle in both injured groups. Since these changes occur without misdirection of regenerating axons, they are interpreted as centrally generated. Sol activity was changed from reciprocal to that of TA in intact rats to coactivate with TA, but only in the Sci group rats. In the T/CF group rats, Sol activity was not altered from that observed in intact rats. Despite effects of injury that limited foot movements, hindlimb kinematics were conserved during downslope walking in both injury groups and during level walking in the T/CF group. During level walking in the Sci group and during upslope walking in both groups of injured rats, the ability to compensate for the
\end{abstract}

effects of the nerve injury was less effective and resulted in longer limb lengths held at more acute angles throughout the step cycle. Changes in limb movements occur irrespective of axon misdirection and reflect compensatory changes in the outputs of the neural circuits that drive locomotion.

Copyright $\odot 2011$ S. Karger AG, Basel

\section{Introduction}

Axons in peripheral nerves form the structural conduit between neuronal circuits in the spinal cord and the musculoskeletal system. They serve as a substrate for both the motor commands to the muscles and for sen-

\section{Abbreviations used in this paper}

EMG electromyographic

MTP metatarsophalangeal

PC principal component

PCA principal components analysis

Sci group sciatic nerve group

Sol soleus

TA tibialis anterior

T/CF group tibial and common fibular nerves group

\section{KARGER}

() 2011 S. Karger AG, Basel

Fax +4161306 1234

E-Mail karger@karger.ch

www.karger.com
Accessible online at: www.karger.com/cto
Dr. Manning J. Sabatier

Department of Natural Sciences, Health and Fitness Management Clayton State University

2000 Clayton State Blvd, Morrow, GA 30260 (USA)

Tel. +1 678466 4619, Fax +1 678466 4669, E-Mail ManningSabatier@ clayton.edu 
sory feedback to the spinal cord. If a peripheral nerve is cut, the relationship between neural circuits and the musculoskeletal system is lost. The vast majority of patients with traumatic peripheral nerve injuries never regain normal function [Brushart, 1998]. One reason given for poor functional outcomes is that axon regeneration in peripheral nerves is slow. Regenerating axons elongate at a rate of only 1-3 mm per day [Fu and Gordon, 1997]. In the proximal stumps of cut nerves they encounter significant inhibition in invading a regeneration pathway in the distal stump. Once they enter this pathway, they encounter potent growth inhibitory molecules [Zuo et al., 1998; Gilbert et al., 2005]. This inhibition delays or prevents early axon regeneration and some axons never participate in the regeneration process.

Another reason for poor functional outcomes following peripheral nerve injuries is the loss of myospecificity resulting from misdirection of regenerating axons [Fawcett and Keynes, 1990]. Although regenerating axons have been found to elongate preferentially through sensory or motor paths [Brushart, 1988, 1993], they do not seem able to distinguish between and/or choose endoneurial tubes leading to their native muscular targets. Therefore, muscles that are downstream of a peripheral nerve injury are commonly reinnervated at least partially by motoneurons that had previously innervated different muscles, sometimes functional antagonists [English, 2005], or by exuberant branches of the same motoneuron that reinnervates two different muscles [Brushart and Mesulam, 1980; Gordon et al., 2003]. This change of myospecificity results in a new relationship between neuronal circuits in the spinal cord and the elements of the musculoskeletal system.

After cutting the sciatic nerves of rats and repairing them with autografts, Gramsbergen et al. [2000] found that the activity patterns of the reinnervated gastrocnemius and tibialis anterior (TA) muscles during locomotion were abnormal. Although they reported no systematic pattern of abnormal activity, coactivation of these antagonist muscles was a common finding. Similar findings were reported from muscles of the hindlimb of mice after common peroneal nerve injury [Wasserschaff, 1990]. Such results could be interpreted as a reflection of the misdirection of regenerating axons described above. Alternatively, such differences in the timing of muscle activation could be due to changes in the outputs of the neural circuits controlling locomotion. Coactivation of agonist and antagonist muscles is thought to increase joint stiffness, and therefore, joint stability [Enoka, 2008]. Coactivation of functional antagonist muscles could be viewed as an adaptation to the new configuration of peripheral axons found following nerve injury. It might be secondary to lower depolarization thresholds and higher afferent-mediated excitatory postsynaptic potentials [Eccles et al., 1958; Gustafsson, 1979; Horch and Lisney, 1981] as well as to consequent hyperreflexia [Valero-Cabre and Navarro, 2001, 2002] which have been observed after peripheral nerve injury.

The goal of this study was to investigate the effect of axon misdirection after sciatic nerve transection on the timing of muscle activity in distal muscle targets and on control of the hindlimb. In addition to changes in muscle activity [Gramsbergen et al., 2000], there are also changes in kinematics [Varejao et al., 2003] during level walking in rats. However, the opportunities for non-level locomotion during normal behavior are abundant. Positive and negative slopes present unique challenges to the nervous system during locomotion [Gregor et al., 2006] and may be useful for characterizing and understanding recovery of function after injury. Therefore, this study examined recovery of muscle activity and limb-level kinematics during downslope, level and upslope locomotion after peripheral nerve injury in rats. We chose to study kinematics of a single vector representation of the hindlimb that has also been adopted in recent studies of cats [Chang et al., 2009] and humans [Ivanenko et al., 2007; Auyang et al., 2009]. This global variable is represented by populations of neurons in the spinal circuits [Bosco and Poppele, 2000; Poppele et al., 2002] and may represent an effort to minimize the complexity of control over a segment that spans multiple joints [Ivanenko et al., 2007]. The use of slope walking in this study, like in a recent study by Maas and colleagues [2010], was essential to characterize the full extent of differences in functional ability that resulted from injury.

\section{Methods}

\section{Procedures}

Adult female Sprague Dawley rats (Rattus norvegicus) weighing 270-315 g were tested in this study. Rats were housed 1 per cage in a temperature- and humidity-controlled room with 12hour light/12-hour dark cycles. They were allowed normal cage activities under standard laboratory conditions and were fed with standard chow and water ad libitum and evaluated daily by clinical veterinarians for signs of discomfort and pain. All procedures were approved by the Institutional Animal Care and Use Committee at Emory University.

Fourteen rats (7 intact, 3 sciatic transection, 2 tibial and common fibular transections, 2 tibial and deep fibular transections) were implanted with wires for electromyographic (EMG) record- 
ing under general anesthesia using sodium pentobarbital (60 mg/ $\mathrm{kg}$ ) or ketamine $(75 \mathrm{mg} / \mathrm{kg})$ and xylazine $(10 \mathrm{mg} / \mathrm{kg})$ administered intraperitoneally and supplemented as needed. Nerve injury and repair was performed at the time of implant for all animals in the injury groups. All surgical procedures were performed under aseptic conditions. Pairs of multi-stranded $(10 \times 50$ gauge, Cooner, AS631) stainless steel fine-wire electrodes stripped of their final $1 \mathrm{~mm}$ of Teflon insulation were implanted in the right soleus (Sol) and TA muscles, as described previously [Hamilton et al., 2010].

In one group of rats, the entire sciatic nerve was transected just proximal to the branch point of the sural nerve (Sci group), using sharp scissors. In another group of rats, the tibial and common fibular nerves were transected separately (T/CF group), each approximately $3 \mathrm{~mm}$ distal to the branch point of the sural nerve. The somata of motoneurons projecting through the tibial and common fibular nerves are grouped into spatially distinct compartments in the spinal cord [Swett et al., 1986] and remain segregated into separate fascicles in the sciatic nerve [Kobbert and Thanos, 2000]. Therefore, this procedure eliminated or at least drastically reduced the probability that regenerating axons from one branch were misdirected into the distal segment of the other. In yet another group of rats, the tibial and deep fibular nerves were transected independently to target axons projecting to the TA specifically and exclude contamination from axons projecting through the common fibular nerve to the peroneus longus, which is active during the stance phase like the Sol [Giroux-Metges et al., 2005]. The common fibular nerve was exposed distal to its insertion into the peroneal muscle layer near the head of the fibula. The epineurium was carefully teased apart and its deep and superficial segments were separated. The deep fibular nerve only was cut with sharp scissors. For all nerve repairs, the proximal and distal segments of the cut nerves were aligned as carefully as possible on a small piece of Gortex and secured in place under very little tension using fibrin glue [Menovsky and Beek, 2001; MacGillivray, 2003; English, 2005].

During the week prior to implantation, each rat was trained to walk on a single-lane plexiglas-enclosed treadmill $(53 \times 10 \times 14$ $\mathrm{cm}$, Columbus Instruments, Columbus, Ohio, USA) intermittently for 3-4 days, 5-10 min/day, and given a Fruit Loop reward. Stress was minimized with low noise levels and gentle handling of the rats in order to help ensure acclimation and to obtain locomotion under normal conditions. Mild intensities of footshock were used as negative reinforcement to improve performance.

\section{Analysis of Functional Recovery}

Locomotor Trials

Hair was closely clipped from around the right hindlimb while the rat was anesthetized with isoflurane. Black marks for digitizing were applied with a permanent marker over the right greater trochanter, lateral malleolus and 5th metatarsophalangeal (MTP) joints to approximate the endpoints of each hindlimb segment. Each rat walked on the treadmill set at $11 \mathrm{~m} / \mathrm{min}$ while it was level $\left(0^{\circ}\right)$ and at two different slope conditions, i.e. $\pm 20^{\circ}( \pm 36.4 \%$ grade), and slope order was randomized. Multiple 15 -second locomotion trials were collected at each of the 3 slopes to ensure that at least 5 step cycles were recorded where the rat was walking at a constant speed. Step cycles were selected for analysis from video records obtained during locomotor trials while the rat was walking in the same location on the belt and had not ridden the belt

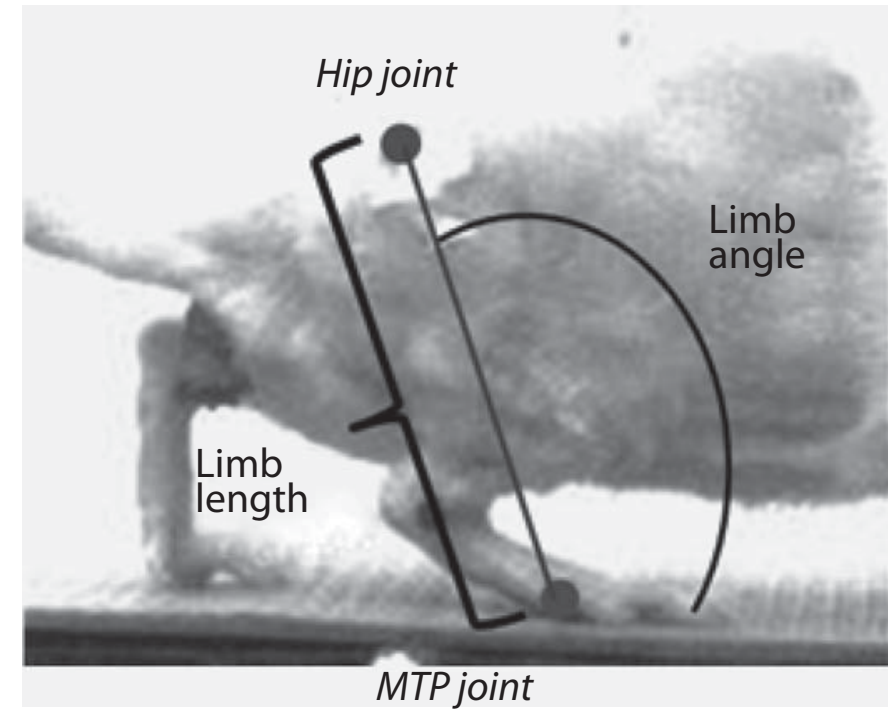

Fig. 1. Diagram of the measurements made. From digitized points over the greater trochanter and the 5th MTP joint, a hindlimb vector was measured from individual video frames sampled at 120 frames/s. The magnitude of this vector is hindlimb length (limb length). Hindlimb angle (limb angle) is formed by the hindlimb vector and the treadmill belt in the rostral direction.

backward or accelerated forward in the preceding step cycles. The plexiglass treadmill enclosure helped control parallax error by minimizing lateral position variation on the treadmill belt.

\section{Kinematics}

Sagittal plane gait kinematics were obtained from a $2 \mathrm{D}$ video of the treadmill locomotion. A single Dragonfly Express highspeed digital camera (Point-Grey Research, Vancouver, B.C., Canada) was used to record motion pictures (120 frames/s) of the right side of the rat while walking on the treadmill. The camera was placed orthogonal to the rat and magnification was calibrated to cover the entire length of the treadmill belt. MaxTRAQ (Innovision Systems, Inc., Columbiaville, Mich., USA) and ImageJ [Rasband, 1997-2006] software were used to digitize the markers over the hip, ankle and MTP joints for each frame of selected step cycles.

From the digitized points, we calculated a single global kinematic variable in each video frame: the vector between the MTP and hip marker positions. The magnitude of this vector is referred to as limb length and the direction is referred to as limb angle, the orientation of the extensible hindlimb to the treadmill belt (fig. 1). These two components of the vector were evaluated to represent overall hindlimb movement during locomotion. We used these estimates of limb length and limb angle in place of individual joint angles (e.g., hip, knee, ankle, MTP joint) because they have been shown to vary much less, both within and between animals, and are accurate representations of the neuromotor control of hindlimb dynamics in locomotion [Bosco and Poppele, 1996; Auyang et al., 2009; Hamilton et al., 2010]. Limb length was normalized in each locomotor trial to the femur length of the animal. 
Femur length was measured for each animal from video frames recorded at each slope and each locomotor trial. This normalization was performed to reduce sources of variations in limb length due to growth of the animal during the course of the study and differences in size between different animals.

\section{Electromyography}

Locomotor EMG activity was recorded from the Sol and TA muscles and synchronized to concurrently collected digitized video with a common pulse. Potentials were amplified $(\times 1,000)$ using a bandpass of $30-1,000 \mathrm{~Hz}$ and then digitized for each 15 -second locomotion trial. Frame numbers corresponding with the onset (paw-on) and termination of step cycles were recorded from videos and then used to extract the corresponding EMG signals. The extracted EMG activity from individual step cycles was rectified and low pass filtered with a time constant of $20 \mathrm{~ms}$, and then time normalized to 100 time bins. Therefore, all EMG figures are expressed as a percentage of the step cycle and have a time scale of 1-100. In the figures in this manuscript, the amplitude of EMG activity has been normalized to the maximum integrated EMG activity recorded from each rat [Gregor et al., 2006; Maas et al., 2010].

\section{Principal Components Analysis}

Principal components analysis (PCA) has been applied to biomechanics [Courtine et al., 2009; Lee et al., 2009] and EMG activity analysis [Widmer et al., 2003; Ivanenko et al., 2004; Stoloff et al., 2007]. It was used here to evaluate differences in locomotor EMG activity profiles between muscles and postdenervation time points. PCA is a technique that offers reduction of a large set of correlated variables to a smaller number of uncorrelated hypothetical components that are termed 'principal components' (PCs) or 'eigenvalues' [Bishop, 1995]. Evaluation of the proportion of overall variance explained by each PC is possible. The first PC necessarily accounts for the largest proportion of the variance in the data (i.e. it has the largest eigenvalue). Each succeeding PC explains the maximum amount of the remaining variance and is uncorrelated to the previous PC. We used this technique to evaluate EMG activity in lieu of other approaches involving subjective determination of discrete events (e.g., onset, duration) in order to focus our analysis on the patterns of muscle activation or the shapes of the EMG activity profiles.

Locomotor EMG activity for each muscle in each rat at each postdenervation time was determined as an average from each of the selected step cycles. The data so obtained were then subjected to PCA. Eigenvalues were obtained using varimax normalization to obtain the maximum variance explained by each PC [Widmer et al., 2003]. PC values were determined for each EMG profile for each PC. Each PC (factor) loading represents the correlation coefficient between the EMG activity profile and the regression line value representing each PC. The PC loadings for each PC were averaged for each muscle at each time point. For each PC studied, mean values were determined for the different muscles at different survival times. Significance of differences in these means was determined using ANOVA, followed by post-hoc paired (Fisher's least significant difference) testing.

\section{Statistical Analysis}

Descriptive statistics were calculated for variables of interest, including aspects of the step cycle, hindlimb kinematics and
Table 1. Average timing of the step cycle during slope walking

\begin{tabular}{|c|c|c|c|}
\hline & Downslope & Level & Upslope \\
\hline $\begin{array}{l}\text { Step cycle duration } \\
\text { Sci group }\end{array}$ & $620 \pm 32$ & $650 \pm 31$ & $710 \pm 25$ \\
\hline 4 weeks & $636 \pm 23$ & $655 \pm 17$ & $741 \pm 9$ \\
\hline 10 weeks & $654 \pm 16$ & $667 \pm 18$ & $801 \pm 17^{*}$ \\
\hline \multicolumn{4}{|l|}{ T/CF group } \\
\hline 4 weeks & $607 \pm 26$ & $640 \pm 68$ & $751 \pm 67$ \\
\hline 10 weeks & $643 \pm 86$ & $697 \pm 40$ & $734 \pm 50$ \\
\hline Stance & $474 \pm 30$ & $504 \pm 24$ & $564 \pm 23$ \\
\hline \multicolumn{4}{|l|}{ Sci group } \\
\hline 4 weeks & $441 \pm 35$ & $457 \pm 33$ & $547 \pm 23$ \\
\hline 10 weeks & $480 \pm 13$ & $482 \pm 24$ & $624 \pm 14$ \\
\hline \multicolumn{4}{|l|}{ T/CF group } \\
\hline 4 weeks & $446 \pm 19$ & $476 \pm 53$ & $608 \pm 55$ \\
\hline 10 weeks & $498 \pm 83$ & $538 \pm 31$ & $607 \pm 40$ \\
\hline Swing & $140 \pm 6$ & $141 \pm 11$ & $141 \pm 6$ \\
\hline \multicolumn{4}{|l|}{ Sci group } \\
\hline 4 weeks & $195 \pm 24^{*}$ & $198 \pm 26^{*}$ & $193 \pm 14^{*}$ \\
\hline 10 weeks & $174 \pm 6^{*}$ & $185 \pm 10^{*}$ & $176 \pm 8^{*}$ \\
\hline \multicolumn{4}{|l|}{$\mathrm{T} / \mathrm{CF}$ group } \\
\hline 4 weeks & $160 \pm 8$ & $164 \pm 17$ & $144 \pm 13$ \\
\hline 10 weeks & $146 \pm 7$ & $159 \pm 14$ & $127 \pm 10$ \\
\hline
\end{tabular}

Values are means \pm SEM, given in $\mathrm{ms}$

${ }^{*} \mathrm{p} \leq 0.05$, significantly different from the value calculated for intact rats at the same slope.

EMG activity. The data are reported as the mean \pm SEM, unless otherwise noted. One-way ANOVA was computed using the mean values for the variables above from all animals to evaluate differences between groups. If the omnibus test was significant, post-hoc tests (Fisher's least significant difference) were computed to determine which pairwise comparisons were significantly different. The significance level was set at 0.05 for all statistical tests.

\section{Results}

\section{Timing of the Step Cycle}

The step cycle in this study was delineated by paw contact with the treadmill belt. We studied the timing of the step cycle in intact rats and in rats during level, downslope and upslope locomotion at different times following transection and surgical repair of the sciatic nerve or its components. These data are summarized in table 1.

Between the different slope groups, changes in both stance and step cycle duration were reported elsewhere [Sabatier et al., 2010]. With only one exception (duration, 


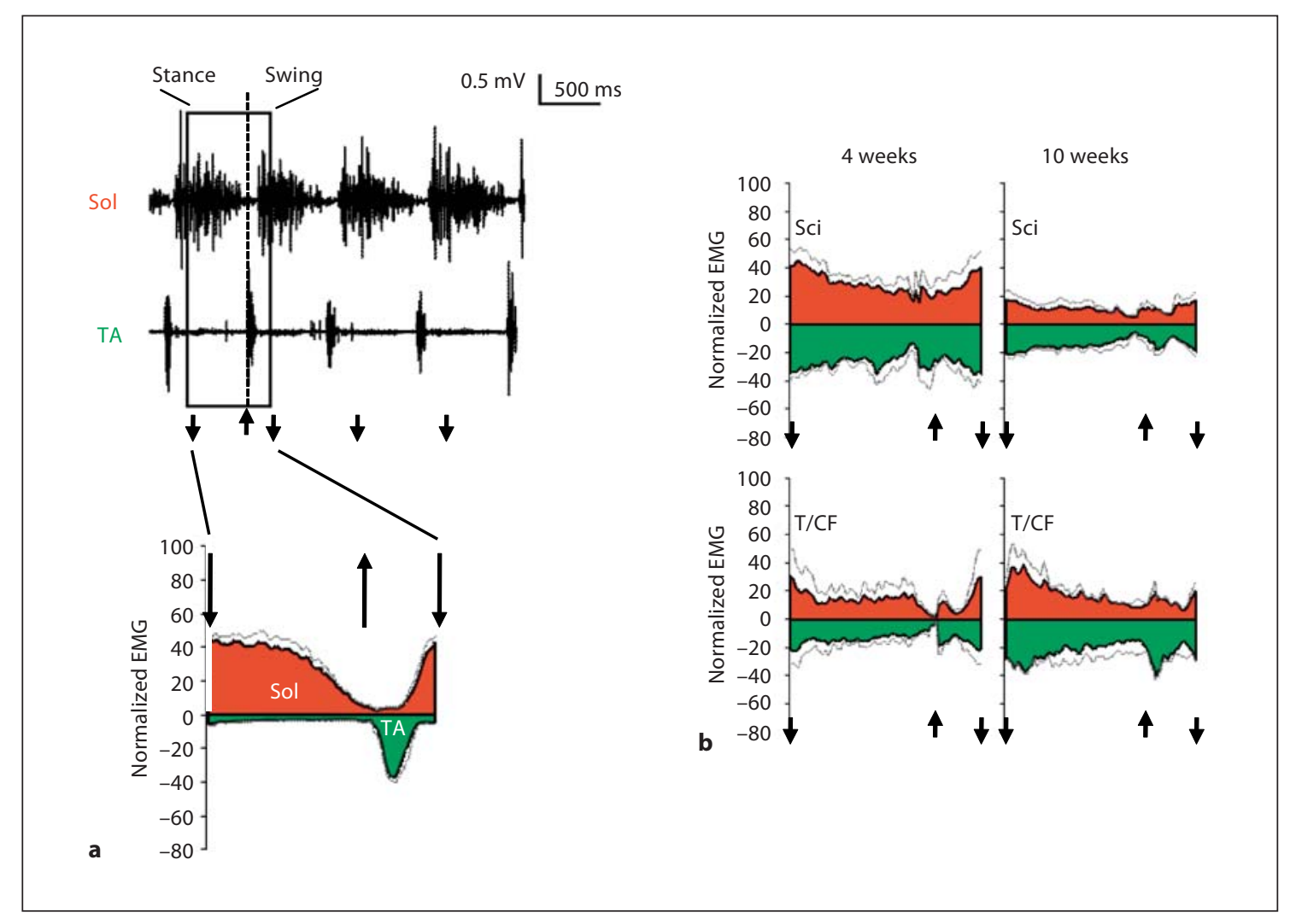

Fig. 2. a Raw EMG activity in Sol and TA muscles during treadmill locomotion. Arrows indicate the times of paw contact $(\downarrow)$ and paw off $(\uparrow)$, based on correlated video records. Below these traces, the average normalized EMG activity of the two muscles during a step cycle is shown. The EMG activity area for the Sol is colored red and that for the TA is colored green. The trace for TA has been inverted. b Average normalized EMG activity is shown for rats at 4 and 10 weeks following transection and surgical repair of the sciatic nerve (Sci) and tibial and common fibular nerves (T/CF).
Sci group, 10 weeks, upslope), at each slope, step cycle duration and stance duration remained the same at different times following nerve transection (table 1). When the branches of the sciatic nerve were cut separately, no significant increase in swing duration was found. In contrast, complete sciatic nerve transection resulted in significant increases in the duration of the swing phase at all 3 slopes studied (table 1). Therefore, all aspects of the timing of the step cycle evaluated in this study were unaffected when the branches of the sciatic nerve were transected separately.

\section{Activity of Reinnervated Muscles}

EMG activity of the Sol and TA muscles was recorded during locomotion to evaluate changes in muscle activation following nerve transection. Four step cycles of typical alternating bursts of Sol and TA EMG activity during level treadmill locomotion at $11 \mathrm{~m} / \mathrm{min}$ are shown at the top of figure 2a. Beneath these raw EMG records are averaged records of rectified and integrated activity from the two muscles during single step cycles. Down and up arrows are used to show the times of paw contact and paw off, respectively. When the sciatic nerve and its branches are intact, activation of these functionally antagonist muscles during locomotion is reciprocal.

Results from animals in whom the tibial and common fibular nerves were cut separately and from animals in whom the tibial and deep fibular nerves were cut separately were indistinguishable. Therefore, in the results that follow, the data from these two groups are collapsed into one group with a sample size of 4 and referred to in the text and figures by the shorthand T/CF. EMG activity in the Sol and TA during treadmill locomotion is synchronous, whether the sciatic nerve was transected completely (fig. 2b, Sci group) or as branches (fig. 2b, T/CF group). The same similarity was found whether record- 
Fig. 3. Analysis of locomotor EMG activity in Sol and TA with PCA. Slope walking PCA factor loadings for TA and Sol from intact animals, injured animals 4 and 10 weeks after transection of the sciatic nerve (Sci) and from animals in whom the tibial and common fibular branches were transected separately (T/CF). Color coding of data from muscles and groups is illustrated. Numbers located near bubbles indicate the postinjury time given in weeks. There is only one data point associated with intact TA and one data point associated with intact Sol in each of the figures (b-d). Therefore, they do not have corresponding numbers. The variance in average rectified and integrated locomotor activity of each muscle was reassigned using PCA. a Scree plot that illustrates the eigenvalues associated with the first 5 PCs. Eigenvalues for the first 2 PCs are represented by stars. bd Average loading values for PC1 and PC2 are illustrated as bubble plots for upslope (Us), downslope (Ds) and level (Lv) walking, respectively. Symbol size is proportional to the SEM (i.e. larger bubbles indicate larger variance about the mean).

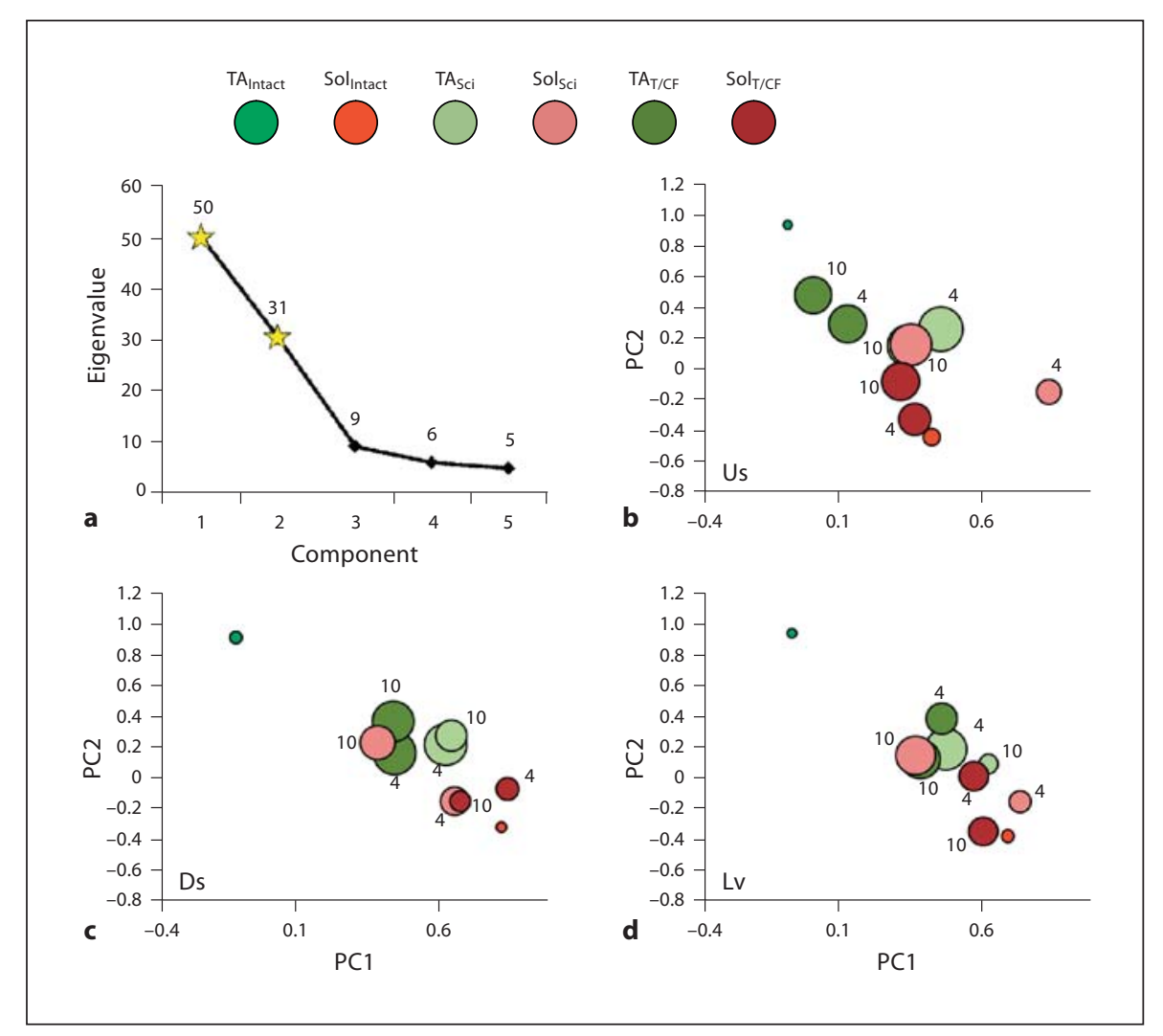

ings were made at 4 weeks after denervation, when initial reinnervation has just begun, or at 10 weeks, when TA and Sol might be expected to be nearly fully reinnervated by regenerating axons. There is both stance and swing phase EMG activity in both muscles for both injury groups at both times studied.

We used PCA to evaluate the relationships between EMG activity profiles of the Sol and TA at different slopes and at different time points in the study. With PCA, the magnitude of the correlations between each EMG activity profile and the overall average EMG activity profile calculated from the entire data set are assigned values called PCs. If EMG activity profiles have similar PC loading values, it is interpreted to mean that their activity profiles are similar to one another.

The proportion of overall variance explained by each $\mathrm{PC}$ is its eigenvalue. Eigenvalues for the first 5 PCs resulting from the PCA are shown in figure 3a. The first PC necessarily counts for the largest proportion of variance in the data (i.e. it has the largest eigenvalue). Each succeeding PC explains the maximum amount of the remaining variance, is uncorrelated to previous $\mathrm{PC}$ and has a smaller eigenvalue. The first two PCs in this study were
50 and 31, respectively. Each eigenvalue thus explains a much larger proportion of overall variance than any single raw variable. Together they explain $68.7 \%$ of the total variance in the data set. The eigenvalue for the third PC explained only $7 \%$ of the variance in the data set. Therefore, only the first two PCs were studied further. Factor (PC) loadings are the correlation between each eigenvalue and the raw data in the set. Average factor loadings for PC1 and PC2 are plotted against one another in figures $3 \mathrm{~b}-\mathrm{d}$ as bubble plots. Results of statistical tests of different comparisons of factor loadings associated with TA and Sol EMG profiles are shown in tables 2 and 3.

For EMG activity in Sol and TA in intact rats, factor loadings are found on opposite corners of the graph for locomotion on each slope (TA, upper left; Sol, lower right). They are significantly different from one another for PC1 and PC2 at each slope. Such distinct differences are consistent with the reciprocal nature of locomotor EMG activity patterns observed in the Sol and TA in intact rats. After nerve transection and repair, and subsequent muscle reinnervation, this large difference between the factor loadings for the two muscles is lost in the Sci group of rats. There are no longer any significant differ- 
Table 2. Comparisons of EMG activity from the TA and Sol in intact rats and both injury groups at each slope

\begin{tabular}{llll}
\hline Group & Ds & Lv & Us \\
\hline $\begin{array}{l}\text { Intact rats } \\
\text { Sci group }\end{array}$ & 1,2 & 1,2 & 1,2 \\
4 weeks & NS & NS & NS \\
10 weeks & NS & NS & NS \\
T/CF group & & & \\
4 weeks & NS & 2 & 2 \\
10 weeks & 2 & 2 & 2 \\
\hline
\end{tabular}

Factor loadings for EMG profiles from the TA versus Sol were compared statistically (least significant difference post-hoc tests).

Ds = Downslope; Lv = level; Us = upslope; 1 = difference in the $\mathrm{PC} 1$ loading is statistically significant ( $\mathrm{p} \leq 0.05) ; 2=$ difference in the PC2 loading is statistically significant ( $\leq \leq 0.05)$; NS = not statistically significant on either factor.

Table 3. Comparisons of TA and Sol EMG activity from postinjury groups versus intact animals

\begin{tabular}{lllll}
\hline Muscle & $\begin{array}{l}\text { Postinjury } \\
\text { time, weeks }\end{array}$ & Ds & Lv & Us \\
\hline TA & & & & \\
$\quad$ Sci group & 4 & 1,2 & 2 & 2 \\
$\quad$ T/CF group & 4 & 2 & 1,2 & 2 \\
$\quad$ Sci group & 10 & 1,2 & 2 & 2 \\
$\quad$ T/CF group & 10 & 2 & 2 & 2 \\
Sol & & & & \\
Sci group & 4 & NS & NS & 1 \\
$\quad$ T/CF group & 4 & NS & 2 & NS \\
$\quad$ Sci group & 10 & 1,2 & 2 & 2 \\
$\quad$ T/CF group & 10 & NS & NS & NS \\
\hline
\end{tabular}

Factor loadings for postinjury EMG profiles from the TA and Sol were compared to factor loadings for EMG profiles of the same muscle from intact animals using the least significant difference post-hoc tests.

$1=$ Difference in the PC1 loading is statistically significant ( $\mathrm{p} \leq 0.05) ; 2=$ difference in the PC2 loading is statistically significant ( $\mathrm{p} \leq 0.05)$; NS = not statistically significant on either factor.

ences in factor loadings between the Sol and TA at any time and/or slope (table 2). We interpret this finding to mean that activity in TA and Sol shifts from reciprocal to coactivation following complete sciatic nerve transection and repair. In contrast, among rats in the T/CF group, significant differences in the factor loadings between TA and Sol are largely preserved. Significant differences in average factor loadings are found in all conditions except downslope at the 4-week sampling point (table 2). We interpret this finding as evidence that the patterns of activation of these two muscles during locomotion remain distinct (although not necessarily the same as found in intact animals, see below) when misdirection of the regenerating axons is reduced or eliminated [Swett et al., 1986; Kobbert and Thanos, 2000].

For both TA and Sol, average factor loadings at different times following nerve transection and repair are more variable than average factor loadings in intact rats, as noted by the larger symbol sizes in figure $3 \mathrm{~b}-\mathrm{d}$. For TA, mean factor loadings at all posttransection times are significantly different from those found in intact rats for at least one factor for all slopes, and irrespective of the transection scheme (Sci or T/CF group; table 3). We interpret this finding as evidence that the pattern of activation of the reinnervated TA muscle differs from that found in intact rats and that this change in activation pattern occurred irrespective of the extent of the misdirection of regenerating axons.

In contrast, average factor loadings for Sol, especially those from the T/CF group, are much more likely not to differ significantly from those found in intact rats. Significant differences were found only during level walking at the 4 -week sampling time (table 3). By 10 weeks after transection, significant differences were found for Sol at all 3 slopes in the Sci group but for none of the slopes for the T/CF group.

\section{Kinematic Analysis}

Adjusted limb length is plotted as a function of limb angle for locomotion at 3 different slopes at 4 and 10 weeks after transection in figure $4 a, b$. The times of paw contact and paw off are marked by open down and up arrows, respectively. Each of the 3 traces in each panel is an average of data from 4 rats. The sizes of the symbols in the graphs are proportional to the SEM. Each tracing follows a counter-clockwise direction. In these graphs, a hysteresis loop is formed, with reduced ordinate values during the swing phase. Adjusted limb length shortens and lengthens during each phase. It decreases more during swing in order for the limb to clear the treadmill belt and bring the foot to its ultimate horizontal position for paw contact. Paw off occurs at the minimal limb angle and paw contact occurs when the limb angle is largest.

Three parameters of these movements were chosen for analysis: limb angle and adjusted limb length at the turning points of the step cycle (paw contact and paw off) and 


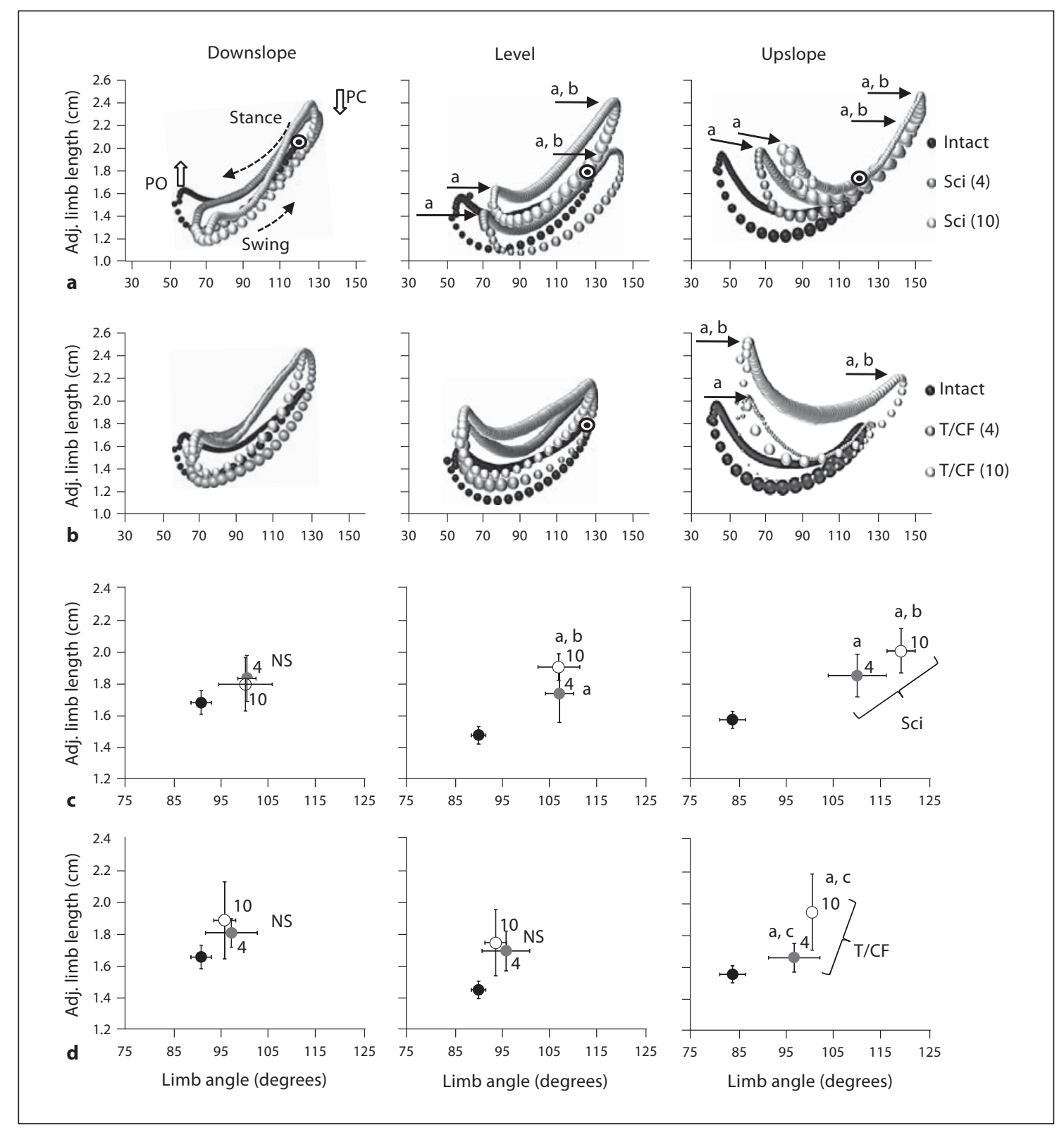

Fig. 4. Hindlimb kinematics during treadmill locomotion. a, b Plots of hindlimb angle (abscissa) versus adjusted (Adj.) hindlimb length (ordinate) are shown for downslope, level and upslope walking. Data from intact rats as well as from rats 4 and 10 weeks following nerve transection in whom the sciatic nerve was transected and repaired (a, Sci group, $n=3$ ) or in whom the tibial and common fibular branches were transected and repaired separately $(\mathbf{b}, \mathrm{T} / \mathrm{CF}$ group, $\mathrm{n}=4$ ). Data from the $\mathrm{T} / \mathrm{CF}$ group rats are always in the foreground. The size of the symbols is proportional to the SEM (i.e. larger bubbles indicate larger variance about the mean). a In the downslope panel, open arrows are next to inflection points in the polygon corresponding to the time at which the paw leaves the treadmill belt (i.e. paw off, PO) and at paw contact (PC) with the treadmill belt. Stance and swing and the direction of the advancement of the step cycle (counter-clockwise) are depicted in this panel as well. a, b Horizontal solid arrows point to maximal femur lengths that were significantly different from intact rats. $\odot=$ Maximal femur lengths from intact rats when postoperative data obscured intact data. c, d Average ( \pm SEM) measures of hindlimb position from the same data are shown; intact and postinjury, Sci group (c), and intact and postinjury, T/CF group (d). ${ }^{\text {a }} \mathrm{p}<0.05$ versus intact rats (angle); ${ }^{\mathrm{b}} \mathrm{p}<0.05$ versus intact rats (length); ${ }^{c} \mathrm{p}<0.05$ versus the Sci group on limb angle at the same postoperation duration. NS $=$ Not significant. 


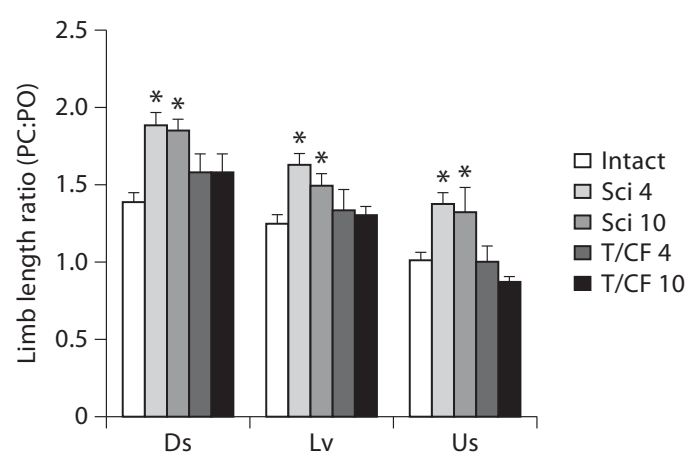

Fig. 5. Limb length ratio at paw contact (PC) and paw off (PO). Average (+SEM) values of the ratio of paw contact:paw off for all groups at each slope are displayed as bar graphs. Asterisks indicate $\mathrm{p}<0.05$ versus intact.

average limb angle and adjusted limb length (fig. 4c, d). There were no significant differences for downslope walking for any of these measures for either transection group. Significant differences in all 3 measures were found at both survival times for rats in the Sci group during level and upslope walking. In these rats, the hindlimbs were held at more acute angles throughout the step cycle and, at least by 10 weeks after transection, adjusted limb length also was longer (fig. 4c). These data are similar to those reported for level walking in sciatic nerve injured rats [Hamilton et al., 2010]. In contrast, significant changes in these kinematic parameters were found for the T/CF groups only during upslope walking (fig. $4 \mathrm{~d}$ ).

The angle-length polygons found in intact rats are rotated clockwise for upslope walking as a result of smaller limb lengths at paw contact and larger limb lengths at paw off, and counter-clockwise for downslope walking as a result of an opposite relationship of the limb lengths at paw contact and paw off (compare solid black symbols in fig. $4 a, b)$. As a measure of this feature, we calculated the ratio of the limb length at paw contact to the limb length at paw off for each animal at each slope. These results are shown in figure 5. A ratio of 1.0 would mean that the limb length was the same at paw contact and paw off. At each slope, the ratio is significantly elevated only for the Sci group. The T/CF group was not significantly different from the intact group at either slope or either time point. Therefore, only T/CF group animals were able to maintain the normal patterns of transition from swing to stance and from stance to swing with respect to control of limb length.

\section{Discussion}

In this study, we evaluated the effect of misdirection of regenerating motor axons on functional recovery by comparing results from 2 different models of denervation and reinnervation of targets of the sciatic nerve. In the Sci group, transection of the entire sciatic nerve was performed. It was assumed in these rats that significant misdirection of regenerating axons into the tibial and common fibular nerves would occur, leading to reinnervation of the Sol and TA muscles by both appropriate and inappropriate motoneurons [Brushart and Mesulam, 1980]. In the second group, the tibial and common fibular branches of the sciatic nerve were cut and repaired separately (T/CF group). It was assumed that in these rats, there would be little or no misdirection of regenerating axons because regenerating axons of the one nerve branch could not reinnervate muscle targets of the other branch in this group. Because we were concerned that the TA muscle might be reinnervated by motoneurons that had once innervated the peroneus longus muscle, which functions slightly differently from TA [Thota et al., 2005], we performed some nerve repairs that excluded the superficial branch of the CF nerve and only included the deep branch, which normally provides innervation to TA. In the end, no significant differences were noted in locomotor EMG activity or hindlimb kinematics between rats in whom the entire common fibular nerve was cut and repaired and rats in whom only the deep branch was cut and repaired.

In both the Sci and T/CF groups, the timing of locomotor EMG activity in the reinnervated TA muscle was strikingly different from that of intact rats. Activity shifted from being restricted to early in the swing phase to nearly the entire step cycle. Because this change occurred irrespective of whether the opportunity for misdirection of regenerating axons was controlled, we suggest that the observed change in timing of TA activity in both groups of rats is not the result of decreased myospecificity of the reinnervated TA muscle resulting from axon misdirection, but the result of a reorganization of the outputs of spinal circuitry. We suggest that this change is a centrally mediated strategy to facilitate control over the distal hindlimb when sensory-motor connections have been disrupted by denervation-reinnervation. An increase in ankle joint stiffness produced by coactivation of TA and Sol during the stance phase might make adjustments in activation of proximal muscles more effective at controlling body weight [Enoka, 2008]. 
Our findings with respect to the timing of locomotor activity in the reinnervated Sol muscle are a little less clear. On visual inspection, reinnervated Sol muscles in both the Sci and T/CF groups appear to change from the pattern observed in intact rats to include significant activity during the swing phase. We would describe the overall changes in TA and Sol as shifting from a strict reciprocal activation to one of marked coactivation. Based on the results of factor analysis using PCs, this observation was supported only for the rats in the Sci group. Factor loadings for Sol for rats in the T/CF group were not significantly different from those of intact rats, suggesting that in reinnervated Sol muscles without contributions from motoneurons that had once innervated targets of the common fibular nerve, any change in activation patterns of Sol could not be detected using PCA. At this time, we cannot determine whether this finding reflects a lack of reorganization of the outputs of spinal circuitry directed at Sol motoneurons when misdirection of reinnervating motoneurons is not found or simply a limitation of our use of PCA to define locomotor activity patterns. What seems clear is that following transection and repair of peripheral nerves, changes in the timing of locomotor activity of reinnervated muscles occur. The sources of this timing are both misdirection of regenerating axons and centrally mediated changes in the outputs of the neural circuits that control locomotion.

Non-level walking constitutes a major portion of the milieu of terrestrial locomotion. Therefore, it is important that some effort is made to include non-level locomotion in the testing battery to ascertain how an animal recovers from a peripheral nerve injury. We incorporated slope walking in this study to challenge the neuromuscular system beyond what is normally encountered during level walking. We have reported on the effects of slope on normal locomotor function (i.e. hindlimb kinematics and EMG activity) in rats elsewhere [Sabatier et al., 2011]. We anticipated that these primarily opposite locomotor challenges might provide additional insight into the recovery of function after peripheral nerve injury. Although we found some slope-related differences in recovery of muscle activity that could be related to differences in the extent of misdirection of regenerating axons, we found more marked differences in hindlimb movements.

We chose to study limb movements in our rats using a global vector of hindlimb length and orientation. Chang and colleagues [2009] have provided convincing evidence that this whole limb approach is powerful in evaluating compensatory changes in limb kinematics following nerve injuries in cats. They showed that even though joint-level kinematics during level walking are significantly compromised after self-reinnervation of the triceps surae in cats, the vector representation of the hindlimb was conserved. Using this method in rats, we have shown [Hamilton et al., 2010], as had Chang et al. [2009] both in cats and in humans [Auyang et al., 2009], that intersubject variability in these global measures was considerably smaller than that of movements of the individual hindlimb joints.

Following complete sciatic nerve transection, several measures of whole limb kinematics were altered during both level and upslope walking. In contrast, movements of the hindlimb were significantly affected only during upslope walking when the branches of the sciatic nerve were transected separately. Even then, the significant changes in limb angle and limb length in the T/CF group of rats during upslope walking were smaller than those found in the Sci group of rats. In both groups of rats, nerve transection markedly affected movements about the ankle joint, both prior to and following muscle reinnervation. The remarkable conservation of both limb angle and limb leng th observed during downslope walking, and also during level walking in the T/CF group rats, reflects effective compensatory movements at the other hindlimb joints, as Chang et al. [2009] have observed for cats. The much more limited ability to conserve these movement parameters during upslope walking in all lesioned rats, and also during level walking in the Sci group rats, is evidence that the compensatory strategy adopted is less effective when walking upslope. The simplest interpretation of these observations is that reinnervation of TA and Sol by functionally inappropriate motor and sensory neurons limits the effectiveness of whatever compensatory mechanisms are being used by the animals to a greater degree than when misdirection of the regenerating axons is prevented. During upslope walking, the stance phase is protracted [Sabatier et al., 2011] and there is increased reliance on force-dependent sensory feedback, specifically related to group Ib afferent activity [Gregor et al., 2006; Donelan et al., 2009]. The extent to which misdirection of force-sensing afferent axons, the motoneuron targets of force-dependent afferent feedback, or both contribute to the force-related locomotor challenges that could not be overcome is not yet known.

It was interesting to find that there were no changes in hindlimb kinematics during downslope walking in either group of injured rats, especially considering the near exact opposite conclusions reached after the study of peripheral nerve injury in cats [Abelew et al., 2000; Maas et al., 2007]. Unfortunately, differences in a number of fea- 
tures of the designs of experiments in our studies and those referenced (e.g., angle of slope, type of injury, species-specific foot posture) make discerning the basis for the differences in results difficult.

To understand the lack of effect on downslope walking that we observed in both groups of lesioned animals, it is important to consider that downslope walking includes more passive components than level or upslope walking. During the late swing phase, hindlimb movements in preparation for paw contact are assisted by gravity. During downslope walking, these passive movements are exaggerated, as the animal must reach farther downward toward a lower contact point on the treadmill belt. Similarly, during the late stance phase, hindlimb extension is truncated in downslope walking, as limb removal is assisted by the inclination of the treadmill belt. Therefore, adapting to downslope walking might be easier for animals with transection injuries of the sciatic nerve or its branches than level or upslope walking.

Following sciatic nerve transection, animals, like human patients, adopt potent compensatory strategies for producing favorable biomechanical outcomes following nerve injuries. Others have reported similar findings in different experimental models [Gordon et al., 1980; Luff and Webb, 1985; Carrier et al., 1997] but not after more subtle insults involving smaller numbers of muscles [Pearson et al., 1999; Misiaszek and Pearson, 2002]. It seems likely that given the large scale of the target denervation found following sciatic nerve transections, compensatory strategies that restore normal level and upslope stepping are simply not possible. It is not entirely clear how animals achieve reasonable locomotion with the new neuromuscular configuration. It likely involves a constellation of changes in function of the proximal muscles [Carrier et al., 1997], resulting in a reduction in knee flexion, movements of the pelvis and movements of the contralateral hindlimb. The nature of the compensatory strategy used may vary considerably from animal to animal or even step cycle to step cycle.

\section{Ackowledgements}

This work was supported by grant HD032571 from the United States Public Health Service. Manning J. Sabatier was supported by the United States Public Health Service National Institutes of Health Institutional Research and Academic Career Development grant, No. K12 GM00680-05.

\section{References}

Abelew, T.A., M.D. Miller, T.C. Cope, T.R. Nichols (2000) Local loss of proprioception results in disruption of interjoint coordination during locomotion in the cat. J Neurophysiol 84: 2709-2714.

-Auyang, A.G., J.T. Yen, Y.H. Chang (2009) Neuromechanical stabilization of leg length and orientation through interjoint compensation during human hopping. Exp Brain Res 192: 253-264.

Bishop, C. (1995) Neural Networks for Pattern Recognition. Oxford, Oxford University Press.

Bosco, G., R.E. Poppele (1996) Temporal features of directional tuning by spinocerebellar neurons: relation to limb geometry. J Neurophysiol 75: 1647-1658.

Bosco, G., R.E. Poppele (2000) Reference frames for spinal proprioception: kinematics based or kinetics based? J Neurophysiol 83: 29462955.

Brushart, T.M. (1988) Preferential reinnervation of motor nerves by regenerating motor axons. J Neurosci 8: 1026-1031.

-Brushart, T.M. (1993) Motor axons preferentially reinnervate motor pathways. J Neurosci 13: $2730-2738$.
Brushart, T.M. (1998) Nerve repair and grafting; in Green, D., R. Hotchkiss, W. Pederson (eds): Green's Operative Hand Surgery. New York, Churchill Livingstone, pp 1381-1403.

- Brushart, T.M., M.M. Mesulam (1980) Alteration in connections between muscle and anterior horn motoneurons after peripheral nerve repair. Science 208: 603-605.

Carrier, L., E. Brustein, S. Rossignol (1997) Locomotion of the hindlimbs after neurectomy of ankle flexors in intact and spinal cats: model for the study of locomotor plasticity. J Neurophysiol 77: 1979-1993.

-Chang, Y.H., A.G. Auyang, J.P. Scholz, T.R. Nichols (2009) Whole limb kinematics are preferentially conserved over individual joint kinematics after peripheral nerve injury. J Exp Biol 212: 3511-3521.

Courtine, G., Y. Gerasimenko, R. van den Brand, A. Yew, P. Musienko, H. Zhong, B. Song, Y. Ao, R.M. Ichiyama, I. Lavrov, R.R. Roy, M.V. Sofroniew, V.R. Edgerton (2009) Transformation of nonfunctional spinal circuits into functional states after the loss of brain input. Nat Neurosci 12: 1333-1342.

Donelan, J.M., D.A. McVea, K.G. Pearson (2009) Force regulation of ankle extensor muscle activity in freely walking cats. J Neurophysiol 101: 360-371.
Eccles, J.C., B. Libet, R.R. Young (1958) The behaviour of chromatolysed motoneurones studied by intracellular recording. J Physiol 143: 11-40.

English, A.W. (2005) Enhancing axon regeneration in peripheral nerves also increases functionally inappropriate reinnervation of targets. J Comp Neurol 490: 427-441.

Enoka, R.M. (2008) Neuromechanics of Human Movement. Champaign, Human Kinetics.

Fawcett, J.W., R.J. Keynes (1990) Peripheral nerve regeneration. Annu Rev Neurosci 13: $43-60$.

Fu, S.Y., T. Gordon (1997) The cellular and molecular basis of peripheral nerve regeneration. Mol Neurobiol 14: 67-116.

Gilbert, R.J., R.J. McKeon, A. Darr, A. Calabro, V.C. Hascall, R.V. Bellamkonda (2005) CS4,6 is differentially upregulated in glial scar and is a potent inhibitor of neurite extension. Mol Cell Neurosci 29: 545-558.

Giroux-Metges, M.A., J.P. Pennec, J. Petit, J. Morel, H. Talarmin, M. Droguet, G. Dorange, M. Gioux (2005) Effects of immobilizing a single muscle on the morphology and the activation of its muscle fibers. Exp Neurol 194: 495-505. 
Gordon, T., J.A. Hoffer, J. Jhamandas, R.B. Stein (1980) Long-term effects of axotomy on neural activity during cat locomotion. J Physiol 303: 243-263.

Gordon, T., O. Sulaiman, J.G. Boyd (2003) Experimental strategies to promote functional recovery after peripheral nerve injuries. J Peripher Nerv Syst 8: 236-250.

Gramsbergen, A., J. Ijkema-Paassen, M.F. Meek (2000) Sciatic nerve transection in the adult rat: abnormal EMG patterns during locomotion by aberrant innervation of hindleg muscles. Exp Neurol 161: 183-193.

-Gregor, R.J., D.W. Smith, B.I. Prilutsky (2006) Mechanics of slope walking in the cat: quantification of muscle load, length change, and ankle extensor EMG patterns. J Neurophysiol 95: 1397-1409.

-Gustafsson, B. (1979) Changes in motoneurone electrical properties following axotomy. J Physiol 293: 197-215.

-Hamilton, S., M. Hinkle, J. Nicolini, L. Rambo, A. Rexwinkle, S. Rose, M. Sabatier, D. Backus, A. English (2010) Misdirection of regenerating axons and functional recovery following sciatic nerve injury in rats. J Comp Neurol 519: 21-33.

Horch, K.W., S.J. Lisney (1981) Changes in primary afferent depolarization of sensory neurones during peripheral nerve regeneration in the cat. J Physiol 313: 287-299.

Ivanenko, Y.P., G. Cappellini, N. Dominici, R.E. Poppele, F. Lacquaniti (2007) Modular control of limb movements during human locomotion. J Neurosci 27: 11149-11161.

-Ivanenko, Y.P., R.E. Poppele, F. Lacquaniti (2004) Five basic muscle activation patterns account for muscle activity during human locomotion. J Physiol 556: 267-282.

Kobbert, C., S. Thanos (2000) Topographic representation of the sciatic nerve motor neurons in the spinal cord of the adult rat correlates to region-specific activation patterns of microglia. J Neurocytol 29: 271-283.
Lee, M., M. Roan, B. Smith (2009) An application of principal component analysis for lower body kinematics between loaded and unloaded walking. J Biomech 42: 2226-2230.

Luff, A.R., S.N. Webb (1985) Electromyographic activity in the cross-reinnervated soleus muscle of unrestrained cats. J Physiol 365: 13-28.

Maas, H., R.J. Gregor, E.F. Hodson-Tole, B.J. Farrell, A.W. English, B.I. Prilutsky (2010) Locomotor changes in length and EMG activity of feline medial gastrocnemius muscle following paralysis of two synergists. Exp Brain Res 203: 681-692.

Maas, H., B.I. Prilutsky, T.R. Nichols, R.J. Gregor (2007) The effects of self-reinnervation of cat medial and lateral gastrocnemius muscles on hindlimb kinematics in slope walking. Exp Brain Res 181: 377-393.

MacGillivray, T.E. (2003) Fibrin sealants and glues. J Card Surg 18: 480-485.

Menovsky, T., J.F. Beek (2001) Laser, fibrin glue, or suture repair of peripheral nerves: a comparative functional, histological, and morphometric study in the rat sciatic nerve. J Neurosurg 95: 694-699.

Misiaszek, J.E., K.G. Pearson (2002) Adaptive changes in locomotor activity following botulinum toxin injection in ankle extensor muscles of cats. J Neurophysiol 87: 229-239.

- Pearson, K.G., K. Fouad, J.E. Misiaszek (1999) Adaptive changes in motor activity associated with functional recovery following muscle denervation in walking cats. J Neurophysiol 82: 370-381.

Poppele, R.E., G. Bosco, A.M. Rankin (2002) Independent representations of limb axis length and orientation in spinocerebellar response components. J Neurophysiol 87: 409422.

Rasband, W.S. (1997-2006) ImageJ. Bethesda, US National Institutes of Health.
Sabatier, M.J., B.N. To, J. Nicolini, A.W. English (2011) Effect of slope and sciatic nerve injury on ankle muscle recruitment and hindlimb kinematics during walking in the rat. J Exp Biol, in press.

Stoloff, R.H., E.P. Zehr, D.P. Ferris (2007) Recumbent stepping has similar but simpler neural control compared to walking. Exp Brain Res 178: 427-438.

Swett, J.E., R.P. Wikholm, R.H. Blanks, A.L. Swett, L.C. Conley (1986) Motoneurons of the rat sciatic nerve. Exp Neurol 93: 227-252.

Thota, A.K., S.C. Watson, E. Knapp, B. Thompson, R. Jung (2005) Neuromechanical control of locomotion in the rat. J Neurotrauma 22: $442-465$.

Valero-Cabre, A., X. Navarro (2001) H reflex restitution and facilitation after different types of peripheral nerve injury and repair. Brain Res 919: 302-312.

-Valero-Cabre, A., X. Navarro (2002) Changes in crossed spinal reflexes after peripheral nerve injury and repair. J Neurophysiol 87: 17631771.

Varejao, A.S., A.M. Cabrita, M.F. Meek, J. BulasCruz, V.M. Filipe, R.C. Gabriel, A.J. Ferreira, S. Geuna, D.A. Winter (2003) Ankle kinematics to evaluate functional recovery in crushed rat sciatic nerve. Muscle Nerve 27: 706-714.

Wasserschaff, M. (1990) Coordination of reinnervated muscle and reorganization of spinal cord motoneurons after nerve transection in mice. Brain Res 515: 241-246.

Widmer, C.G., D.I. Carrasco, A.W. English (2003) Differential activation of neuromuscular compartments in the rabbit masseter muscle during different oral behaviors. Exp Brain Res 150: 297-307.

Zuo, J., Y.J. Hernandez, D. Muir (1998) Chondroitin sulfate proteoglycan with neurite-inhibiting activity is up-regulated following peripheral nerve injury. J Neurobiol 34: 4154. 